\title{
ON THE STRUCTURE OF THE LOWER EDGE OF THE SPECTRUM OF THE PERIODIC MAGNETIC SCHRÖDINGER OPERATOR WITH SMALL MAGNETIC POTENTIAL
}

\author{
R. G. SHTERENBERG
}

In fond memory of Ol'ga Aleksandrovna Ladyzhenskaya

\begin{abstract}
For the periodic magnetic Schrödinger operator, the structure of the lower edge of the spectrum is investigated. It is known that in the nonmagnetic case the energy depends quadratically on the quasimomentum in the neighborhood of the lower edge of the spectrum. Herewith, the operator admits a convenient "multiplicative" factorization, which makes it possible to investigate the threshold effects efficiently. It is shown that for sufficiently small magnetic potential the magnetic Schrödinger operator also admits a similar factorization.
\end{abstract}

\section{$\S 0$. INTRODUCTION}

0.1. Let $\Gamma$ be a lattice in $\mathbb{R}^{d}, d \geq 1$, and let $\Omega$ be an elementary cell of the lattice $\Gamma$. It is known (see, e.g., $\underline{\mathrm{Sk}}$ ) that the $\Gamma$-periodic differential operators (DOs) can be partially diagonalized with the help of the Gelfand transformation. Then the initial DO is represented as a direct integral of a family of DOs that depend on a parameter $\mathbf{k} \in \mathbb{R}^{d}$ (called the quasimomentum) and act on the torus associated with $\Omega$.

We consider lower semibounded selfadjoint DOs. For most of the DOs occurring in mathematical physics, the spectrum of the associated operators acting in $L_{2}(\Omega)$ is discrete. Let $E_{j}(\mathbf{k}), j=1,2, \ldots$, be the corresponding eigenvalues arranged in nondecreasing order. The functions $E_{j}(\mathbf{k})$ depend on $\mathbf{k}$ continuously. Then the spectrum of the initial DO has a band structure. The bands coincide with the images of the band functions $E_{j}(\cdot)$. It is convenient to assume that the lower edge of the spectrum is $\lambda=0$.

It turns out that the solution of certain questions only requires the knowledge of the structure of the spectrum lower edge. In such cases we talk about threshold effects. One of the brightest examples of a threshold effect is the homogenization problem, i.e., the study of the behavior of a periodic DO in the small period limit.

0.2. In $[\mathrm{BSu}$, a general method was developed to investigate threshold effects for operators admitting a convenient ("regular") factorization. In the scalar case this factorization is described by the following condition: the operator $M$ in question admits a representation in the form

$$
M=\overline{f^{-1}(\mathbf{x})} \mathbf{b}(\mathbf{D})^{*} G(\mathbf{x}) \mathbf{b}(\mathbf{D}) f^{-1}(\mathbf{x}), \quad \mathbf{b}(\mathbf{D}):=\sum_{j=1}^{d} D_{j} \mathbf{b}_{j},
$$

2000 Mathematics Subject Classification. Primary 35J10, 35P15.

Key words and phrases. Periodic operator, magnetic Schrödinger operator, lower edge of the spectrum, threshold effects, factorization.

Supported by RFBR (grant no. 02-01-00798). 
where $\mathbf{D}=\operatorname{col}\left\{D_{1}, \ldots, D_{d}\right\}=-i \nabla=-i \operatorname{col}\left\{\frac{\partial}{\partial x_{1}}, \ldots, \frac{\partial}{\partial x_{d}}\right\}, f(\mathbf{x})$ is a $\Gamma$-periodic function, $G(\mathbf{x})$ is a $\Gamma$-periodic $(m \times m)$-matrix-valued function $(m \geq d)$, and $\mathbf{b}_{j}, j=$ $1,2, \ldots, d$, is a set of linearly independent vectors in $\mathbb{C}^{m}$. Furthermore,

$$
f, f^{-1} \in L_{\infty}\left(\mathbb{R}^{d}\right) ; \quad c_{0} \mathbf{1} \leq G(\mathbf{x}) \leq c_{1} \mathbf{1}, \mathbf{x} \in \mathbb{R}^{d}, 0<c_{0} \leq c_{1}<\infty .
$$

Note that the existence of such a factorization directly implies the so-called "proper" structure of the lower edge of the spectrum. This means the following:

1) the first band function $E_{1}(\mathbf{k})$ has a nondegenerate minimum at the point $\mathbf{k}=0$ (and $E_{1}(0)=0$ );

2) we have $E_{1}(\mathbf{k})>0, \mathbf{k} \neq 0(\bmod \Gamma)$;

3) $\min _{\mathbf{k}} E_{2}(\mathbf{k})>0$.

Sometimes, the DO to be dealt with is not given directly in the form (0.1), but can be reduced to it. It was noted in $[\mathrm{BSu}$ that, in particular, the periodic Schrödinger operator $-\operatorname{div} g(\mathbf{x}) \nabla+V(\mathbf{x})$ with a positive matrix-valued function (metric) $g(\mathbf{x})$ admits a factorization as in (0.1). The same is true for the two-dimensional periodic Pauli operator $(\mathbf{D}-\mathbf{A})^{2} \pm\left(\partial_{1} A_{2}-\partial_{2} A_{1}\right)$, which is a particular case of the magnetic Schrödinger operator

$$
(\mathbf{D}-\mathbf{A})^{*} g(\mathbf{x})(\mathbf{D}-\mathbf{A})+V(\mathbf{x}) .
$$

Here $\mathbf{A}(\mathbf{x})$ is a $\Gamma$-periodic $\mathbb{R}^{2}$-valued function.

0.3. What was said above justifies the interest in a description of other classes of operators representable as in (0.1). In the present paper we investigate the operator (0.3). The main results are Theorem 2.7 and Theorem 2.8, where we prove the existence of a factorization (0.1) for the operator (0.3) with sufficiently small magnetic potential $\mathbf{A}(\mathbf{x})$. In $[\mathrm{Sh}$ it was shown that, without the smallness assumption, the operator (0.3) may fail to admit factorization as in (0.1), and moreover, the lower edge of the spectrum may fail to have a proper structure.

0.4. Acknowledgements. My interest in the topics considered in this paper was raised by the papers of M. Sh. Birman and T. A. Suslina, to whom I am sincerely grateful for fruitful discussions. Also, I thank A. A. Arkhipova for consultations concerning properties of the solutions of elliptic equations.

\section{$\S 1$. Notation}

1.1. We use the notation

$$
\begin{aligned}
& \mathbf{x}=\operatorname{col}\left(x_{1}, x_{2}, \ldots, x_{d}\right) \in \mathbb{R}^{d} \\
& \nabla=\nabla_{\mathbf{x}}=\operatorname{col}\left(\partial / \partial x_{1}, \partial / \partial x_{2}, \ldots, \partial / \partial x_{d}\right) \\
& \mathbf{D}=\operatorname{col}\left(D_{1}, D_{2}, \ldots, D_{d}\right)=-i \nabla \\
& \text { Next, }\langle\cdot, \cdot\rangle,|\cdot| \text { are the standard inner product and the norm in } \mathbb{C}^{d} .
\end{aligned}
$$

Let $\Gamma$ be a lattice of periods in $\mathbb{R}^{d}$. We denote by $\Omega$ an elementary cell of the lattice $\Gamma$ and by $\bar{\Omega}$ the closure of $\Omega$. The Sobolev classes of order 1 with integrability index $p$ are denoted by $W_{p}^{1}\left(\mathbb{R}^{d}\right), W_{p}^{1}(\Omega)$; for $p=2$ we abbreviate this to $H^{1}\left(\mathbb{R}^{d}\right), H^{1}(\Omega)$. The subspace formed by the functions in $W_{p}^{1}(\Omega)$, the $\Gamma$-periodic extension of which belongs to $W_{p \text {,loc }}^{1}\left(\mathbb{R}^{d}\right)$, is denoted by $\widetilde{W}_{p}^{1}(\Omega)$; for $p=2$ we use the notation $\widetilde{H}^{1}(\Omega)$. The set of restrictions to $\Omega$ of the $\Gamma$-periodic functions in $\mathbb{R}^{d}$ that are Hölder of order $\delta$ is denoted by $\widetilde{C}^{\delta}(\Omega), \delta \in(0,1)$.

Next, $\|\cdot\|_{\mathcal{B}}$ is the norm in a Banach space $\mathcal{B}$. By $c, C$ (with or without indices) we denote various constants in the estimates. By 1 we denote the unit $(d \times d)$-matrix. 
1.2. We consider the operator

$$
M=M(g, \mathbf{A}, V):=(\mathbf{D}-\mathbf{A}(\mathbf{x}))^{*} g(\mathbf{x})(\mathbf{D}-\mathbf{A}(\mathbf{x}))+V(\mathbf{x}), \quad \mathbf{x} \in \mathbb{R}^{d},
$$

in the space $L_{2}\left(\mathbb{R}^{d}\right), d \geq 2$. All coefficients are assumed to be real-valued and $\Gamma$-periodic. Also, we assume that the following conditions are fulfilled:

$$
\begin{gathered}
V \in L_{p / 2}(\Omega), \quad p>d, \\
\mathbf{A} \in L_{p}(\Omega), \quad p>d, \\
c_{0} \mathbf{1} \leq g(\mathbf{x}) \leq c_{1} \mathbf{1} \quad \text { for a.e. } \mathbf{x} \in \mathbb{R}^{d}, 0<c_{0} \leq c_{1}<\infty .
\end{gathered}
$$

Under conditions (1.2)-(1.4), the operator $M$ is given via the closed lower semibounded form

$$
\begin{aligned}
m[u, u]= & m(g, \mathbf{A}, V)[u, u] \\
:= & \int_{\mathbb{R}^{d}}\langle g(\mathbf{x})(\mathbf{D}-\mathbf{A}(\mathbf{x})) u(\mathbf{x}),(\mathbf{D}-\mathbf{A}(\mathbf{x})) u(\mathbf{x})\rangle d \mathbf{x} \\
& +\int_{\mathbb{R}^{d}} V(\mathbf{x})|u(\mathbf{x})|^{2} d \mathbf{x}, \quad u \in H^{1}\left(\mathbb{R}^{d}\right) .
\end{aligned}
$$

The periodic operator $M$ can be expanded in the direct integral of a family of operators $M(\mathbf{k}), \mathbf{k} \in \widetilde{\Omega}(\widetilde{\Omega}$ is an elementary cell of the dual lattice $\widetilde{\Gamma})$. The operator $M(\mathbf{k}), \mathbf{k} \in \mathbb{R}^{d}$, is defined in $L_{2}(\Omega)$ via the closed lower semibounded form

$$
\begin{aligned}
m(\mathbf{k})[u, u]= & m(\mathbf{k} ; g, \mathbf{A}, V)[u, u] \\
:= & \int_{\Omega}\langle g(\mathbf{x})(\mathbf{D}-\mathbf{A}(\mathbf{x})+\mathbf{k}) u(\mathbf{x}),(\mathbf{D}-\mathbf{A}(\mathbf{x})+\mathbf{k}) u(\mathbf{x})\rangle d \mathbf{x} \\
& +\int_{\Omega} V(\mathbf{x})|u(\mathbf{x})|^{2} d \mathbf{x}, \quad u \in \widetilde{H}^{1}(\Omega) .
\end{aligned}
$$

The spectrum of $M(\mathbf{k})$ is discrete. Let $\left\{E_{j}(\mathbf{k})\right\}$ denote the eigenvalues of the $M(\mathbf{k})$, enumerated in nondecreasing order:

$$
E_{1}(\mathbf{k}) \leq E_{2}(\mathbf{k}) \leq \cdots \leq E_{n}(\mathbf{k}) \leq \cdots .
$$

Let $\left\{\phi_{j}(\mathbf{x}, \mathbf{k})\right\}$ be the corresponding (periodic) eigenfunctions orthonormal in $L_{2}(\Omega)$. In what follows, all functions defined on $\Omega$ are assumed to be extended to $\mathbb{R}^{d} \Gamma$-periodically. The functions $E_{j}(\mathbf{k})$ are continuous and $\widetilde{\Gamma}$-periodic. We have

$$
\sigma(M)=\bigcup_{j} \mathcal{R}\left(E_{j}(\mathbf{k})\right), \quad \mathbf{k} \in \mathbb{R}^{d} .
$$

Here the symbol $\mathcal{R}(\cdot)$ stands for the range of a function.

\section{$\S 2$. FACTORIZATION}

2.1. We fix a triplet $\{\lambda, \mathbf{k}, f\}$ :

$$
\lambda \in \mathbb{R}, \quad \mathbf{k} \in \mathbb{R}^{d}, \quad 0 \neq f \in \widetilde{H}^{1}(\Omega) .
$$

Definition 2.1. Assume that conditions (1.2)-(1.4) are satisfied. We say that the differential expression (1.1) admits a factorization $F(\lambda, \mathbf{k}, f)$ if, for the given triplet (2.1), there exists a $\Gamma$-periodic selfadjoint matrix-valued function $G \in L_{1, \text { loc }}\left(\mathbb{R}^{d}\right)$ such that the identity

$$
\overline{f(\mathbf{x})} e^{-i\langle\mathbf{k}, \mathbf{x}\rangle}\left((\mathbf{D}-\mathbf{A}(\mathbf{x}))^{*} g(\mathbf{x})(\mathbf{D}-\mathbf{A}(\mathbf{x}))+V(\mathbf{x})-\lambda\right) e^{i\langle\mathbf{k}, \mathbf{x}\rangle} f(\mathbf{x})=\mathbf{D}^{*} G(\mathbf{x}) \mathbf{D}
$$

is fulfilled in the sense of distributions. 
Definition 2.2. We say that the factorizations $F\left(\lambda_{1}, \mathbf{k}_{1}, f_{1}\right)$ and $F\left(\lambda_{2}, \mathbf{k}_{2}, f_{2}\right)$ are equivalent if $\lambda_{1}=\lambda_{2}, \mathbf{k}_{1}-\mathbf{k}_{2} \in \widetilde{\Gamma}$, and $f_{2}(\mathbf{x})=\alpha e^{i\left\langle\left(\mathbf{k}_{1}-\mathbf{k}_{2}\right), \mathbf{x}\right\rangle} f_{1}(\mathbf{x})$ for some $\alpha \in \mathbb{C} \backslash\{0\}$.

It is easy to understand that if a factorization $F(\lambda, \mathbf{k}, f)$ exists, then a selfadjoint matrix $G(\mathbf{x})$ satisfying (2.2) is not unique. The set of all $\Gamma$-periodic selfadjoint matrixvalued functions $G \in L_{1, \text { loc }}\left(\mathbb{R}^{d}\right)$ satisfying $(2.2)$ is denoted by $\mathcal{G}(\lambda, \mathbf{k}, f)$.

If in addition to (2.1) we assume that the functions $f$ and $f^{-1}$ are multipliers in $H^{1}\left(\mathbb{R}^{d}\right)$ (then so they are also in $\widetilde{H}^{1}(\Omega)$ ), then $(2.2)$ implies that $M(\mathbf{k}) f=\lambda f$. Therefore, to construct a factorization of the form (2.2), we shall act as follows.

Let $\lambda \in \sigma(M)$. Then, by (1.7), there exists a quasimomentum $\mathbf{k}$ and a function $0 \neq f \in \widetilde{H}^{1}(\Omega)$ such that

$$
M(\mathbf{k}) f=\lambda f .
$$

By using (2.3), it is easy to verify that (2.2) is fulfilled if and only if

$$
G(\mathbf{x})=g(\mathbf{x})|f(\mathbf{x})|^{2}+i J(\mathbf{x}),
$$

where $J(\mathbf{x})$ is a real-valued $\Gamma$-periodic antisymmetric matrix-valued function belonging to $L_{1, \text { loc }}\left(\mathbb{R}^{d}\right)$ and satisfying

$$
(\operatorname{div} J(\mathbf{x}))^{t}=-2|f(\mathbf{x})|^{2} g(\mathbf{x})(\mathbf{A}(\mathbf{x})-\mathbf{k})+2 \operatorname{Im} \overline{f(\mathbf{x})} g(\mathbf{x}) \nabla f(\mathbf{x})=: \eta(\mathbf{x} ; \lambda, \mathbf{k}, f)
$$

in the sense of distributions. It is well known that equation (2.5) is solvable in the class of real antisymmetric periodic matrix-valued functions if and only if the following conditions are satisfied:

$$
\begin{gathered}
\operatorname{div} \eta(\mathbf{x} ; \lambda, \mathbf{k}, f)=0 \\
\int_{\Omega} \eta(\mathbf{x} ; \lambda, \mathbf{k}, f) d \mathbf{x}=0 .
\end{gathered}
$$

It is easily seen that (2.6) is a direct consequence of (2.3). Thus, the existence problem for the factorization $F(\lambda, \mathbf{k}, f)$ reduces to verification of $(2.7)$.

Theorem 2.3. Let

$$
\lambda_{0}:=\min \sigma(M) .
$$

Suppose that $\mathbf{k}_{0} \in \mathbb{R}^{d}$ and $0 \neq f_{0} \in \widetilde{H}^{1}(\Omega)$ satisfy a relation of the form (2.3):

$$
M\left(\mathbf{k}_{0}\right) f_{0}=\lambda_{0} f_{0} .
$$

Then the factorization $F\left(\lambda_{0}, \mathbf{k}_{0}, f_{0}\right)$ exists.

Proof. The above constructions show that it suffices to check identity (2.7), where $\eta$ is defined as in (2.5) with $\lambda_{0}, \mathbf{k}_{0}, f_{0}$ in the role of $\lambda, \mathbf{k}, f$. Identities (1.7) and (2.8) imply

$$
M(\mathbf{k})-\lambda_{0} I \geq 0, \quad \mathbf{k} \in \mathbb{R}^{d} .
$$

Here the symbol $I$ stands for the identity operator. In particular, (2.10) means that

$$
m(\mathbf{k})\left[f_{0}, f_{0}\right] \geq \lambda_{0}\left\|f_{0}\right\|^{2}, \quad \mathbf{k} \in \mathbb{R}^{d} .
$$

We put $\mathbf{k}:=\mathbf{k}_{0}+\kappa$ in (2.11) and use (2.9). Then (2.11) can be rewritten as follows (we use the notation (2.5)):

$$
\int_{\Omega}\left\langle\eta\left(\mathbf{x} ; \lambda_{0}, \mathbf{k}_{0}, f_{0}\right), \kappa\right\rangle d \mathbf{x}+\int_{\Omega}\langle g(\mathbf{x}) \kappa, \kappa\rangle\left|f_{0}(\mathbf{x})\right|^{2} d \mathbf{x} \geq 0, \quad \kappa \in \mathbb{R}^{d} .
$$

Obviously, from (1.4) it follows that the last inequality is equivalent to (2.7). 
Remark 2.4. By similar arguments, it can be proved that the factorization (2.2) exists for some other points $\lambda \in \sigma(M)$ provided that the quasimomentum $\mathbf{k}$ and the function $f$ are chosen appropriately. In particular, there are factorizations with respect to the edges of the internal gaps of the spectrum of $M$ (if such gaps exist). However, this fact is not important for us now.

Theorem 2.3 shows that the factorization (2.2) exists. However, relation (2.2) is rather weak and does not contain sufficient information.

Definition 2.5. Suppose there exists a factorization $F(\lambda, \mathbf{k}, f)$, and let $\mathcal{G}(\lambda, \mathbf{k}, f)$ be the corresponding family of matrices $G(\mathbf{x})$ satisfying $(2.2)$.

1) We say that the family of matrices $\mathcal{G}(\lambda, \mathbf{k}, f)$ is positive if it contains at least one matrix $G(\mathbf{x})$ satisfying

$$
c_{0}^{\prime} \mathbf{1} \leq G(\mathbf{x}) \leq c_{1}^{\prime} \mathbf{1} \text { for a.e. } \mathbf{x} \in \mathbb{R}^{d}, 0<c_{0}^{\prime} \leq c_{1}^{\prime}<\infty .
$$

2) We say that the factorization $F(\lambda, \mathbf{k}, f)$ is proper if

a) the family $\mathcal{G}(\lambda, \mathbf{k}, f)$ is positive;

b) the functions $f$ and $f^{-1}$ are multipliers in $H^{1}\left(\mathbb{R}^{d}\right)$.

Obviously, equivalent factorizations are proper or not proper simultaneously.

Let $F(\lambda, \mathbf{k}, f)$ be a proper factorization, and let $G(\mathbf{x})$ be a representative of the corresponding positive family $\mathcal{G}(\lambda, \mathbf{k}, f)$ satisfying (2.12). Obviously, the form

$$
m_{F}[u, u]:=\int_{\mathbb{R}^{d}}\left\langle G(\mathbf{x}) \mathbf{D}\left(f^{-1}(\mathbf{x}) u(\mathbf{x})\right), \mathbf{D}\left(f^{-1}(\mathbf{x}) u(\mathbf{x})\right)\right\rangle d \mathbf{x}, \quad u \in H^{1}\left(\mathbb{R}^{d}\right),
$$

is closed and lower semibounded. The form (2.13) gives rise to a selfadjoint operator $M_{F}$ in $L_{2}\left(\mathbb{R}^{d}\right)$. Then, relation $(2.2)$ means that

$$
e^{-i\langle\mathbf{k}, \mathbf{x}\rangle}(M-\lambda I) e^{i\langle\mathbf{k}, \mathbf{x}\rangle}=M_{F} .
$$

Note that the form $m_{F}$ and the operator $M_{F}$ do not depend on a representative $G(\mathbf{x})$ satisfying (2.12) (this follows, e.g., from (2.14)). Relation (2.14) implies the following elementary lemma.

Lemma 2.6. If a proper factorization $F(\lambda, \mathbf{k}, f)$ exists, then this factorization is equivalent to the factorization $F\left(\lambda_{0}, \mathbf{k}_{0}, f_{0}\right)$ constructed in Theorem 2.3. In particular, we have

$$
E_{1}(\mathbf{k})>\lambda_{0} \text { for } \mathbf{k}-\mathbf{k}_{0} \notin \widetilde{\Gamma} ; \quad E_{2}(\mathbf{k})>\lambda_{0}, \mathbf{k} \in \mathbb{R}^{d} .
$$

Proof. This is an easy consequence of (1.7) and (2.12)-(2.14).

2.2. It is well known that a proper factorization exists if $\mathbf{A}(\mathbf{x})=0$. In $[\mathrm{Sh}]$ it was shown that, generally speaking, for a nontrivial magnetic potential, this is not the case anymore. Nevertheless, for sufficiently small magnetic potentials a proper factorization still exists.

Consider an operator of the form (1.1), where now we write $t \mathbf{A}(\mathbf{x})$ in place of $\mathbf{A}(\mathbf{x})$. Here, $\mathbf{A}(\mathbf{x})$ is a $\Gamma$-periodic magnetic potential satisfying (1.3), and $t \in \mathbb{R}$ is the coupling constant. We shall use the notation introduced above, indicating explicitly the dependence of all objects on the parameter $t$. For purely technical reasons, we strengthen the conditions on the metric in the case of dimensions $d \geq 3$. We assume that for some $\delta \in(0,1)$ we have

$$
g \in \widetilde{C}^{\delta}(\Omega), \quad \delta \in(0,1) .
$$

Theorem 2.7. Suppose that $d=2$ and conditions (1.2)-(1.4) are satisfied. Let $M_{t}$ be the operator defined via the form (1.5) with $t \mathbf{A}(\mathbf{x}), t \in \mathbb{R}$, in place of $\mathbf{A}(\mathbf{x})$. Then for sufficiently small $|t|$ a proper factorization $F\left(\lambda_{0}(t), \mathbf{k}_{0}(t), f_{0}(\mathbf{x} ; t)\right)$ exists. 
Theorem 2.8. Suppose that $d \geq 3$ and conditions (1.2)-(1.4), (2.16) are satisfied. Let $M_{t}$ be the operator defined via the form (1.5) with $t \mathbf{A}(\mathbf{x}), t \in \mathbb{R}$, in place of $\mathbf{A}(\mathbf{x})$. Then for sufficiently small $|t|$ a proper factorization $F\left(\lambda_{0}(t), \mathbf{k}_{0}(t), f_{0}(\mathbf{x} ; t)\right)$ exists.

Proof of Theorem 2.7. As above, let

$$
\lambda_{0}(t):=\min \sigma\left(M_{t}\right), \quad t \in \mathbb{R},
$$

and let $\mathbf{k}_{0}(t)$ be a quasimomentum satisfying

$$
\mathbf{k}_{0}(t) \in \widetilde{\Omega}_{B}, \quad E_{1}\left(\mathbf{k}_{0}(t), t\right)=\lambda_{0}(t) .
$$

Here, $\widetilde{\Omega}_{B}$ is an open Brillouin zone (recall that $0 \in \widetilde{\Omega}_{B}$ ). The standard perturbation theory yields

$$
\mathbf{k}_{0}(t) \rightarrow 0 \quad \text { as } t \rightarrow 0 .
$$

The form $m_{t}(\mathbf{k})$ extends to arbitrary $\{\mathbf{k}, t\} \in \mathbb{C}^{d} \times \mathbb{C}$ analytically. The form $m_{t}(\mathbf{k})$ is closed and sectorial. Such a form gives rise (see [K] Theorems VI.2.1, 2.5, 2.7]) to an $m$-sectorial operator, still denoted by $M_{t}(\mathbf{k})$. The resolvent of the operator $M_{t}(\mathbf{k})$, $\{\mathbf{k}, t\} \in \mathbb{C}^{d} \times \mathbb{C}$, is compact.

Since the eigenvalue $E_{1}(0,0)$ is simple, the standard analytic perturbation theory (see $[\mathrm{K}]$ ) shows that there exists a neighborhood $\mathcal{U}_{0} \subset \mathbb{C}^{d} \times \mathbb{C}$ of the point $\{\mathbf{k}=0, t=0\}$ such that the eigenvalue $E_{1}(\mathbf{k}, t)$ of the operator $M_{t}(\mathbf{k})$ is simple and depends on $\{\mathbf{k}, t\}$ analytically in $\mathcal{U}_{0}$. Moreover, the eigenfunction corresponding to the eigenvalue $E_{1}(\mathbf{k}, t)$ can also be chosen to be analytic in $\mathcal{U}_{0}$ and normal in $\mathcal{U}_{0} \cap\left\{\mathbb{R}^{d} \times \mathbb{R}\right\}$. We denote such an eigenfunction by $f_{1}(\mathbf{x} ; \mathbf{k}, t)$. It is well known (see, e.g., [LaU]) that for $\mathbf{k}=0, t=0$ the first eigenfunction of the operator $M_{0}(0)$ can be chosen to be strictly positive. Therefore, there is no loss of generality in assuming that a phase factor of the function $f_{1}(\mathbf{x} ; \mathbf{k}, t)$ is fixed in such a way that

$$
f_{1}(\mathbf{x} ; 0,0) \geq c_{2}>0 .
$$

Taking (2.19) into account, in what follows we assume that the parameter $|t|(t \in \mathbb{R})$ is chosen so small that

$$
\left\{\mathbf{k}_{0}(t), t\right\} \in \mathcal{U}_{0} \cap\left\{\mathbb{R}^{d} \times \mathbb{R}\right\} .
$$

Then we put

$$
f_{0}(\mathbf{x} ; t):=f_{1}\left(\mathbf{x} ; \mathbf{k}_{0}(t), t\right), \quad t \in \mathbb{R} .
$$

Initially, the function $f_{1}(\mathbf{x} ; \mathbf{k}, t)$ is only $\widetilde{H}^{1}(\Omega)$-analytic in $\mathcal{U}_{0}$. However, for some $q>2$ we have (see, e.g., $[\mathrm{BeFr}$ )

$$
f_{1}(\cdot ; \mathbf{k}, t) \in \widetilde{W}_{q}^{1}(\Omega)(\subset \widetilde{C}(\Omega)),
$$

and the estimate

$$
\left\|f_{1}(\cdot ; \mathbf{k}, t)\right\|_{W_{q}^{1}(\Omega)} \leq C, \quad\{\mathbf{k}, t\} \in \mathcal{U}_{0},
$$

is fulfilled uniformly in $\mathcal{U}_{0}$.

By using the Cauchy formula, it is not difficult to check that estimate (2.23) and the $\widetilde{H}^{1}(\Omega)$-analyticity of the function $f_{1}$ imply the $\widetilde{W}_{q}^{1}(\Omega)$-analyticity of $f_{1}$ in $\mathcal{U}_{0}$. In particular, combining this with (2.19) and (2.21), we see that

$$
\max _{\mathbf{x} \in \bar{\Omega}}\left|f_{0}(\mathbf{x} ; t)-f_{1}(\mathbf{x} ; 0,0)\right| \rightarrow 0 \quad \text { as } t \rightarrow 0 .
$$

By (2.20) and (2.24), for sufficiently small $|t|$ we obtain

$$
\left|f_{0}(\mathbf{x} ; t)\right| \geq c_{2} / 2>0 \text {. }
$$


Relations (2.21), (2.22), and (2.25) imply that for sufficiently small $|t|$ condition b) of Definition 2.5 is satisfied. Thus, to prove that the factorization $F\left(\lambda_{0}(t), \mathbf{k}_{0}(t), f_{0}(\mathbf{x} ; t)\right)$ is proper we only need to check that the family $\mathcal{G}\left(\lambda_{0}(t), \mathbf{k}_{0}(t), f_{0}(\mathbf{x} ; t)\right)$ is positive. In accordance with the proof of Theorem 2.3, the matrices $G$ in the family $\mathcal{G}$ obey the identity (see (2.4))

$$
G(\mathbf{x})=g(\mathbf{x})\left|f_{0}(\mathbf{x} ; t)\right|^{2}+i J(\mathbf{x} ; t),
$$

where the real antisymmetric periodic matrix $J(\mathbf{x} ; t)$ solves the equation (see $(2.5)$ )

$$
(\operatorname{div} J(\mathbf{x} ; t))^{t}=-2\left|f_{0}(\mathbf{x} ; t)\right|^{2} g(\mathbf{x})\left(t \mathbf{A}(\mathbf{x})-\mathbf{k}_{0}(t)\right)+2 \operatorname{Im} \overline{f_{0}(\mathbf{x} ; t)} g(\mathbf{x}) \nabla f_{0}(\mathbf{x} ; t)=: \eta_{t}(\mathbf{x}) .
$$

From Theorem 2.3 we know that

$$
\operatorname{div} \eta_{t}(\mathbf{x})=0, \quad \int_{\Omega} \eta_{t}(\mathbf{x}) d \mathbf{x}=0
$$

so that equation (2.27) is solvable. Moreover, since the function $f_{1}(\mathbf{x} ; \mathbf{k}, t)$ is $\widetilde{W}_{q}^{1}(\Omega)$ analytic and $f_{1}(\mathbf{x} ; 0,0)$ is real-valued (see $\left.(2.20)\right)$, relation (2.19) implies

$$
\eta_{t} \in L_{q}(\Omega), \quad\left\|\eta_{t}\right\|_{L_{q}(\Omega)} \rightarrow 0 \text { as } t \rightarrow 0 .
$$

Using (2.28), (2.29), and the Marcinkiewicz theorem (see [Ma]), we see that there is a solution of equation (2.27) such that

$$
J(\cdot ; t) \in \widetilde{W}_{q}^{1}(\Omega)(\subset \widetilde{C}(\Omega)), \quad\|J(\cdot ; t)\|_{W_{q}^{1}(\Omega)} \rightarrow 0 \text { as } t \rightarrow 0 .
$$

Relations (1.4), (2.25), (2.26), and (2.30) show that for sufficiently small $|t|$ the family $\mathcal{G}\left(\lambda_{0}(t), \mathbf{k}_{0}(t), f_{0}(\mathbf{x} ; t)\right)$ has a representative that satisfies $(2.12)$; hence, the family $\mathcal{G}\left(\lambda_{0}(t), \mathbf{k}_{0}(t), f_{0}(\mathbf{x} ; t)\right)$ is positive.

Proof of Theorem 2.8 is quite similar to the proof of Theorem 2.7. We mention some differences. We use the following statement (see [Tr, Theorem 3.7]).

Theorem 2.9. Suppose $d \geq 2$ and conditions (1.4), (2.16) are satisfied. Let $\mathbf{h}(\mathbf{x})$ be a vector-valued function belonging to $L_{s}(\Omega)$, and let $h(\mathbf{x})$ be a scalar-valued function belonging to $L_{s / 2}(\Omega)$ for some $s \in(d, \infty)$. Moreover, assume that $\int_{\Omega} h(\mathbf{x}) d \mathbf{x}=0$. Then, in the sense of distributions on the torus, the equation

$$
\operatorname{div} g(\mathbf{x}) \nabla u(\mathbf{x})=\operatorname{div} \mathbf{h}(\mathbf{x})+h(\mathbf{x})
$$

admits a solution such that $\int_{\Omega} u(\mathbf{x}) d \mathbf{x}=0$, and we have

$$
\begin{gathered}
u \in \widetilde{W}_{s}^{1}(\Omega), \\
\|u\|_{W_{s}^{1}(\Omega)} \leq C(\Omega, g, s)\left(\|\mathbf{h}\|_{L_{s}(\Omega)}+\|h\|_{L_{s / 2}(\Omega)}\right) .
\end{gathered}
$$

Suppose that $\varphi(\mathbf{x} ; \mathbf{k}, t)$ solves the equation

$$
\operatorname{div} g(\mathbf{x}) \nabla \varphi(\mathbf{x} ; \mathbf{k}, t)=\operatorname{div} g(\mathbf{x})(t \mathbf{A}(\mathbf{x})-\mathbf{k})
$$

on the torus under the additional condition $\int_{\Omega} \varphi(\mathbf{x} ; \mathbf{k}, t) d \mathbf{x}=0$. Then, by (1.3) and Theorem 2.9,

$$
\varphi(\cdot ; \mathbf{k}, t) \in \widetilde{W}_{p}^{1}(\Omega)(\subset \widetilde{C}(\Omega))
$$


R. G. SHTERENBERG

Moreover, the function $\varphi(\mathbf{x} ; \mathbf{k}, t)$ is linear with respect to $\mathbf{k}$ and $t$. Thus, for the gauge magnetic potential

$$
\widetilde{\mathbf{A}}(\mathbf{x} ; \mathbf{k}, t):=t \mathbf{A}(\mathbf{x})-\mathbf{k}-\nabla \varphi(\mathbf{x} ; \mathbf{k}, t)
$$

we have

$$
\begin{gathered}
\widetilde{\mathbf{A}} \in L_{p}(\Omega) \\
\operatorname{div} g(\mathbf{x}) \widetilde{\mathbf{A}}(\mathbf{x} ; \mathbf{k}, t)=0 .
\end{gathered}
$$

Put

$$
u(\mathbf{x} ; \mathbf{k}, t):=e^{-i \varphi(\mathbf{x} ; \mathbf{k}, t)} f_{1}(\mathbf{x} ; \mathbf{k}, t) .
$$

Then

$$
\left((\mathbf{D}-\widetilde{\widetilde{\mathbf{A}}(\mathbf{x} ; \mathbf{k}, t)})^{*} g(\mathbf{x})(\mathbf{D}-\widetilde{\mathbf{A}}(\mathbf{x} ; \mathbf{k}, t))+V(\mathbf{x})\right) u(\mathbf{x} ; \mathbf{k}, t)=E_{1}(\mathbf{k}, t) u(\mathbf{x} ; \mathbf{k}, t) .
$$

The function $u$ is $\widetilde{H}^{1}(\Omega)$-analytic in $\mathcal{U}_{0}$. Relations (1.2), (1.4), (2.36), and (2.39) imply (see [LaU]) that $u \in \widetilde{C}(\Omega)$, and moreover, the following estimate is fulfilled uniformly in $\mathcal{U}_{0}$ :

$$
\|u(\cdot ; \mathbf{k}, t)\|_{C(\Omega)} \leq C, \quad\{\mathbf{k}, t\} \in \mathcal{U}_{0} .
$$

Thus (cf. above) $u$ is $\widetilde{C}(\Omega)$-analytic.

Next, by (2.37) and (2.39) we have

$$
\begin{aligned}
\operatorname{div} g(\mathbf{x}) \nabla u(\mathbf{x} ; \mathbf{k}, t)= & 2 i \operatorname{div} g(\mathbf{x}) \widetilde{\mathbf{A}}(\mathbf{x} ; \mathbf{k}, t) u(\mathbf{x} ; \mathbf{k}, t) \\
& +V(\mathbf{x}) u(\mathbf{x} ; \mathbf{k}, t)-E_{1}(\mathbf{k}, t) u(\mathbf{x} ; \mathbf{k}, t) \\
& +\langle g(\mathbf{x}) \widetilde{\mathbf{A}}(\mathbf{x} ; \mathbf{k}, t), \widetilde{\widetilde{\mathbf{A}}(\mathbf{x} ; \mathbf{k}, t)\rangle u(\mathbf{x} ; \mathbf{k}, t) .}
\end{aligned}
$$

Then, from (1.2), (1.4), (2.16), (2.21), (2.35), (2.36), (2.38), (2.40), the $\widetilde{C}(\Omega)$-analyticity of the function $u$ and Theorem 2.9 , we deduce that

$$
f_{1}(\cdot ; \mathbf{k}, t) \in \widetilde{W}_{p}^{1}(\Omega)
$$

with the same $p>d(p<\infty)$ as in (1.2) and (1.3) and that, moreover,

$$
\left\|f_{1}(\cdot ; \mathbf{k}, t)\right\|_{W_{p}^{1}(\Omega)} \leq C, \quad\{\mathbf{k}, t\} \in \mathcal{U}_{0}
$$

uniformly in $\mathcal{U}_{0}$. Thus, the function $f_{1}$ is $\widetilde{W}_{p}^{1}(\Omega)$-analytic in $\mathcal{U}_{0}$.

The rest of the proof repeats the proof of Theorem 2.7 with $p$ in place of $q$.

\section{REFERENCES}

[BSu] M. Sh. Birman and T. A. Suslina, Second order periodic differential operators. Threshold properties and homogenization, Algebra i Analiz 15 (2003), no. 5, 1-108; English transl., St. Petersburg Math. J. 15 (2004), no. 5, 639-714. MR2068790 (2005k:47097)

[BeFr] A. Bensoussan and J. Frehse, Regularity results for nonlinear elliptic systems and applications, Appl. Math. Sci., vol. 151, Springer-Verlag, Berlin, 2002. MR1917320(2004a:31001)

[K] T. Kato, Perturbation theory for linear operators, Springer-Verlag, Berlin, 1995. MR1335452 (96a:47025)

[LaU] O. A. Ladyzhenskaya and N. N. Ural'tseva, Linear and quasilinear equations of elliptic type, 2nd ed., "Nauka", Moscow, 1973; English transl. of 1st ed., Acad. Press, New York-London, 1968. MR0509265 (58:23009) MR0244627 (39:5941)

[Ma] J. Marcinkiewicz, Sur les multiplicateurs des series de Fourier, Studia Math. 8 (1939), 78-91.

[Sh] R. G. Shterenberg, An example of a periodic magnetic Schrödinger operator with a degenerate lower edge of the spectrum, Algebra i Analiz 16 (2004), no. 2, 177-185; English transl., St. Petersburg Math. J. 16 (2005), no. 2, 417-422. MR2068347 (2005d:35220) 
[Sk] M. M. Skriganov, Geometric and arithmetic methods in the spectral theory of multidimensional periodic operators, Trudy Mat. Inst. Steklov. 171 (1985), 122 pp.; English transl., Proc. Steklov Inst. Math. 1987, no. 2. MR0798454 (87h:47110)

[Tr] G. M. Troianiello, Elliptic differential equations and obstacle problems, Univ. Ser. Math., Plenum Press, New York, 1987. MR.1094820 (92b:35004)

Department of Physics, St. Petersburg State University, Ulyanovskaya 1, Petrodvorets, St. Petersburg 198504, Russia

E-mail address: roman@RS3759.spb.edu

Received 28/FEB/2005

Translated by THE AUTHOR 